Manuscript Number: 5078R3

Title: A new mammalian model system for thalidomide teratogenesis: Monodelphis domestica

Article Type: SI: Dev-Angio

Keywords: teratogen; congenital birth defects; limb; angiogenesis

Corresponding Author: Dr. Karen E Sears, PhD

Corresponding Author's Institution: University of Illinois

First Author: Daniel Sorensen

Order of Authors: Daniel Sorensen; Amanda Sackett; Daniel J Urban; Jennifer Maier; Neil Vargesson; Karen E Sears, PhD

Manuscript Region of Origin: USA

Abstract: From 1957 to 1962, thalidomide caused birth defects in >10,000 children. While the drug was pulled from the market, thalidomide is currently prescribed to treat conditions including leprosy. As a result, a new generation of babies with thalidomide defects is being born in the developing world. This represents a serious problem, as the mechanisms by which thalidomide disrupts development remain unresolved. This lack of resolution is due, in part, to the absence of an appropriate mammalian model for thalidomide teratogenesis. We test the hypothesis that opossum (Monodelphis domestica) is well suited to model human thalidomide defects. Results suggest that opossum embryos exposed to thalidomide display a range of phenotypes (e.g., heart, craniofacial, limb defects) and penetrance similar to humans. Furthermore, all opossums with thalidomide defects exhibit vascular disruptions. Results therefore support the hypotheses that opossums make a good mammalian model for thalidomide teratogenesis, and that thalidomide can severely disrupt angiogenesis in mammals. 


\begin{abstract}
From 1957 to 1962 , thalidomide caused birth defects in $>10,000$ children. While the drug was pulled from the market, thalidomide is currently prescribed to treat conditions including leprosy. As a result, a new generation of babies with thalidomide defects is being born in the developing world. This represents a serious problem, as the mechanisms by which thalidomide disrupts development remain unresolved. This lack of resolution is due, in part, to the absence of an appropriate mammalian model for thalidomide teratogenesis. We test the hypothesis that opossum (Monodelphis domestica) is well suited to model human thalidomide defects. Results suggest that opossum embryos exposed to thalidomide display a range of phenotypes (e.g., heart, craniofacial, limb defects) and penetrance similar to humans. Furthermore, all opossums with thalidomide defects exhibit vascular disruptions. Results therefore support the hypotheses that opossums make a good mammalian model for thalidomide teratogenesis, and that thalidomide can severely disrupt angiogenesis in mammals.
\end{abstract}




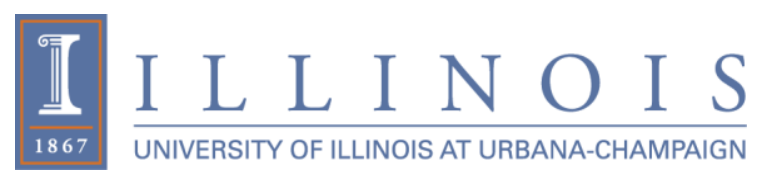

Dept. of Animal Biology, School of Integrative Biology Program in Ecology, Evolution and Conservation Biology Institute for Genomic Biology

January 17,2017

Editor-in-Chief

Reproductive Toxicology

Dear Editor-in-Chief

We are writing to respectfully request that you consider our revised manuscript, "A new mammalian model system for thalidomide teratogenesis: Monodelphis domestica" for publication in Reproductive Toxicology. The manuscript is being submitted for the upcoming special issue on development and angiogenesis.

This study describes a new mammalian model for thalidomide defects in humans, the opossum (Monodelphis domestica). This represents an important step forward for the field that fills a critical gap in the field as, until now, there has not been an appropriate mammalian model for thalidomide teratogenesis in humans. Opossum embryos exposed to thalidomide display a range of phenotypes (e.g., heart, craniofacial, limb defects) and penetrance similar to humans, and opossums have larger litter sizes and are easy to breed in the lab. We were able to address all of the new reviewer comments (see below for details). We are thankful for the comments, and making the suggested changes improved the manuscript.

Given its scope and relevance, this manuscript should be of interest to scientists with interests in teratology, thalidomide, developmental biology, and mammalian systems. As such, we are confident that Reproductive Toxicology readers will find this manuscript to be of general interest. Thank you for your consideration, and please feel free to contact us should you have any questions regarding our submission.

Sincerely,

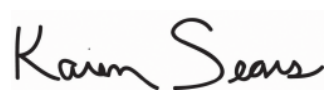

465 Morrill Hall, 505 South Goodwin Avenue, Urbana IL 61801

Cell: 217-898-3011, Email: ksears@life.illinois.edu, http://www.life.uiuc.edu/sears 
1. The authors continue to make statement or draw conclusions from data not in evidence in their paper. Specifically, in the second paragraph of the second full page of the Discussion the authors state "Given that opossums routinely have litters containing 8-15 embryos..." followed in the first line of the next paragraph which reads: Given the opossum's large litters..." Table 1 of their data does not support these statements. In a total of 12 litters in this study, the mean number of embryos per litter is $7.5 ; 6 / 12$ of the litters had $\leq 6$ embryos only 4 litters had $\geq 10$ embryos per litter. Consequently, the first statement need a literature reference to support it and a brief discussion as to why this set of litters was so small. In addition, the second statement might be reworded to state: "Given the opossum's litter size..." leaving out any subjective judgment as to size.

KES: We have changed the language to reflect our findings in this study. "Given that the opossums from this study had an average of 7.5 embryos per litter, this means that most thalidomide-treated litters will likely produce many embryos displaying the "mildly deformed" phenotype and at least 1 embryo with the "severely deformed" phenotype. In line with this, all treated litters in our study generated several "mildly deformed" and at least 1 "severely deformed" embryo." And "Given the opossum's litter size, high penetrance of thalidomideinduced defects, and defects similar to humans, this study strongly supports the hypothesis that the gray short-tailed opossum..."

2. In the aforementioned paragraph, the discussion of the opossum placenta is weak or incorrect in several ways. First, the opossum has a choriovitelline placenta (which is not true of the eutherian mammals used in safety research - they possess inverted yolk sac placentae, which are quite different). Second, the choriovitelline placenta does not invade the uterine wall in any way similar to the invasions experienced by rodents or humans. Third, the lines that appear to equate "formation of placenta" in rodents or humans to opossums seems weak. It does appear that in their model thalidomide gets to the embryo by means of the choriovitelline placenta, but this is a strictly a diffusion driven model as opposed to the hemochorial arrangement in rodents and humans. Thus, this statement would needs to be qualified.

KES: We have altered the text to read, "Furthermore, while the reproductive process of opossums, as marsupial mammals, does differ from that of humans and other placental mammals in some ways (e.g., choriovitelline placenta in marsupials vs. hemochorial placenta in humans), both marsupial and placental mammals share internal development, a reproductive trait critical to the process of teratogenesis. This similarity represents a significant advantage of the opossum model, relative to in vitro and non-mammalian models."

3. The last paragraph beginning at the bottom of the second page of the Discussion states: "The results of this study also strongly support the hypothesis that thalidomide can severely disrupt angiogenesis in mammals." However, consideration of the new data and figures added to the paper need to be incorporated into the paragraph. In the last paragraph of the Results, the authors note "midbrain blood pooling" in a control embryo. The authors need to deal with this finding in the Discussion. Certainly these data appear to weaken the statement about "strongly" supporting their hypothesis. I believe their hypothesis may have merit, but they need to provide the reader with rationale as to how this control embryo relates to their hypothesis. 
KES: We have removed the "strongly" from the discussion, and generally tempered our discussion language. We have also edited the text to the following, "The results of this study also support the hypothesis that thalidomide can severely disrupt angiogenesis in mammals. All embryos displaying a thalidomide-induced phenotype, be they "mildly deformed" or "severely deformed", exhibited clear angiogenic defects. These defects ranged from hemorrhages and/or blood pooling in "mildly deformed" embryos to a complete vascular disruption in some "severely deformed" embryos. Moreover, while one of control embryo exhibits some isolated blood pooling, and thus some evidence for angiogenic disruption, the range (i.e., structures impacted) and magnitude (i.e., degree of severity) of disruptions displayed by treatment embryos is greater. These findings are therefore consistent with the hypothesis that thalidomide induces defects in mammals, including humans, at least in part by disrupting proper angiogenesis $[21,22$, 51]."

4. Finally, the authors need to emphasize the preliminary nature of their data in their conclusions. The addition of control litters led to one litter with 2 severely malformed embryos. The veracity of this new model will require more historical control data being gathered - the authors are commended for starting this process and (potentially) sparking interest among other investigators.

KES: We thank the reviewer for their comments. We have edited the final paragraph to the following, "To confirm the results obtained from the limited trials in this study, additional thalidomide and control trials should be performed in the opossum system. However, given the opossum's litter size, high penetrance of thalidomide-induced defects, and defects similar to humans, results of this initial study support the hypothesis that the gray short-tailed opossum (Monodelphis domestica) makes a good mammalian model system for thalidomide-induced deformities in humas. In particular, the opossum system seems to provide a good opportunity to investigate the hypothetical role of vascular disruption in thalidomide teratogenesis... We are following up on the research presented here by performing additional thalidomide and control trials in opossum, and detailed analyses of the cellular and molecular processes that are disrupted by thalidomide exposure in the opossum model." 
1. The authors continue to make statement or draw conclusions from data not in evidence in their paper. Specifically, in the second paragraph of the second full page of the Discussion the authors state "Given that opossums routinely have litters containing 8-15 embryos..." followed in the first line of the next paragraph which reads: Given the opossum's large litters..." Table 1 of their data does not support these statements. In a total of 12 litters in this study, the mean number of embryos per litter is $7.5 ; 6 / 12$ of the litters had $\leq 6$ embryos only 4 litters had $\geq 10$ embryos per litter. Consequently, the first statement need a literature reference to support it and a brief discussion as to why this set of litters was so small. In addition, the second statement might be reworded to state: "Given the opossum's litter size..." leaving out any subjective judgment as to size.

KES: We have changed the language to reflect our findings in this study. "Given that the opossums from this study had an average of 7.5 embryos per litter, this means that most thalidomide-treated litters will likely produce many embryos displaying the "mildly deformed" phenotype and at least 1 embryo with the "severely deformed" phenotype. In line with this, all treated litters in our study generated several "mildly deformed" and at least 1 "severely deformed" embryo." And "Given the opossum's litter size, high penetrance of thalidomideinduced defects, and defects similar to humans, this study strongly supports the hypothesis that the gray short-tailed opossum..."

2. In the aforementioned paragraph, the discussion of the opossum placenta is weak or incorrect in several ways. First, the opossum has a choriovitelline placenta (which is not true of the eutherian mammals used in safety research - they possess inverted yolk sac placentae, which are quite different). Second, the choriovitelline placenta does not invade the uterine wall in any way similar to the invasions experienced by rodents or humans. Third, the lines that appear to equate "formation of placenta" in rodents or humans to opossums seems weak. It does appear that in their model thalidomide gets to the embryo by means of the choriovitelline placenta, but this is a strictly a diffusion driven model as opposed to the hemochorial arrangement in rodents and humans. Thus, this statement would needs to be qualified.

KES: We have altered the text to read, "Furthermore, while the reproductive process of opossums, as marsupial mammals, does differ from that of humans and other placental mammals in some ways (e.g., choriovitelline placenta in marsupials vs. hemochorial placenta in humans), both marsupial and placental mammals share internal development, a reproductive trait critical to the process of teratogenesis. This similarity represents a significant advantage of the opossum model, relative to in vitro and non-mammalian models."

3. The last paragraph beginning at the bottom of the second page of the Discussion states: "The results of this study also strongly support the hypothesis that thalidomide can severely disrupt angiogenesis in mammals." However, consideration of the new data and figures added to the paper need to be incorporated into the paragraph. In the last paragraph of the Results, the authors note "midbrain blood pooling" in a control embryo. The authors need to deal with this finding in the Discussion. Certainly these data appear to weaken the statement about "strongly" supporting their hypothesis. I believe their hypothesis may have merit, but they need to provide the reader with rationale as to how this control embryo relates to their hypothesis. 
KES: We have removed the "strongly" from the discussion, and generally tempered our discussion language. We have also edited the text to the following, "The results of this study also support the hypothesis that thalidomide can severely disrupt angiogenesis in mammals. All embryos displaying a thalidomide-induced phenotype, be they "mildly deformed" or "severely deformed", exhibited clear angiogenic defects. These defects ranged from hemorrhages and/or blood pooling in "mildly deformed" embryos to a complete vascular disruption in some "severely deformed" embryos. Moreover, while one of control embryo exhibits some isolated blood pooling, and thus some evidence for angiogenic disruption, the range (i.e., structures impacted) and magnitude (i.e., degree of severity) of disruptions displayed by treatment embryos is greater. These findings are therefore consistent with the hypothesis that thalidomide induces defects in mammals, including humans, at least in part by disrupting proper angiogenesis $[21,22$, 51]."

4. Finally, the authors need to emphasize the preliminary nature of their data in their conclusions. The addition of control litters led to one litter with 2 severely malformed embryos. The veracity of this new model will require more historical control data being gathered - the authors are commended for starting this process and (potentially) sparking interest among other investigators.

KES: We thank the reviewer for their comments. We have edited the final paragraph to the following, "To confirm the results obtained from the limited trials in this study, additional thalidomide and control trials should be performed in the opossum system. However, given the opossum's litter size, high penetrance of thalidomide-induced defects, and defects similar to humans, results of this initial study support the hypothesis that the gray short-tailed opossum (Monodelphis domestica) makes a good mammalian model system for thalidomide-induced deformities in humas. In particular, the opossum system seems to provide a good opportunity to investigate the hypothetical role of vascular disruption in thalidomide teratogenesis... We are following up on the research presented here by performing additional thalidomide and control trials in opossum, and detailed analyses of the cellular and molecular processes that are disrupted by thalidomide exposure in the opossum model." 
-Thalidomide-exposed opossums display phenotypes including severe blood pooling and hemorrhaging, limb truncations, digit losses, heart defects, and mandibular truncations. -Several thalidomide-induced phenotypes in opossums are similar to those observed in thalidomide-exposed humans, and thalidomide defects are similarly penetrant in opossums and humans.

-All opossums with thalidomide defects exhibit vascular disruptions, consistent with thalidomide being a disrupter of blood vessel formation.

-Phenotypic results of this study suggest that opossums might make a good mammalian model for thalidomide teratogenesis, and set the stage for further thalidomide research in opossum. 


\title{
A new mammalian model system for thalidomide teratogenesis: Monodelphis domestica
}

\author{
Daniel Sorensen ${ }^{1}$, Amanda Sackett ${ }^{1}$, Daniel J. Urban ${ }^{1}$, Jennifer Maier ${ }^{1}$, \\ Neil Vargesson ${ }^{2}$, and Karen E. Sears ${ }^{1,3 *}$
}

${ }^{1}$ School of Integrative Biology, 505 South Goodwin Avenue, University of Illinois, Urbana IL USA 61801

${ }^{2}$ School of Medicine, Medical Sciences and Nutrition. Institute of Medical Sciences. University of Aberdeen. Foresterhill. Aberdeen. AB25 2ZD. UK.

${ }^{3}$ Institute for Genomic Biology, 1206 W Gregory Drive, University of Illinois, Urbana IL USA 61801

*Corresponding Author:

Dr. Karen E. Sears, School of Integrative Biology, University of Illinois, 465 Morrill Hall, 505

South Goodwin Avenue, Urbana IL

Email: ksears@life.illinois.edu; Phone: 217-244-7855

Running head: Thalidomide teratogenesis in opossums

Keywords: teratogen, congenital birth defects, limb, angiogenesis 


\begin{abstract}
From 1957 to 1962, thalidomide caused birth defects in $>10,000$ children. While the drug was pulled from the market, thalidomide is currently prescribed to treat conditions including leprosy. As a result, a new generation of babies with thalidomide defects is being born in the developing world. This represents a serious problem, as the mechanisms by which thalidomide disrupts development remain unresolved. This lack of resolution is due, in part, to the absence of an appropriate mammalian model for thalidomide teratogenesis. We test the hypothesis that opossum (Monodelphis domestica) is well suited to model human thalidomide defects. Results suggest that opossum embryos exposed to thalidomide display a range of phenotypes (e.g., heart, craniofacial, limb defects) and penetrance similar to humans. Furthermore, all opossums with thalidomide defects exhibit vascular disruptions. Results therefore support the hypotheses that opossums make a good mammalian model for thalidomide teratogenesis, and that thalidomide can severely disrupt angiogenesis in mammals.
\end{abstract}




\section{Introduction}

Thalidomide was originally marketed in 1957 as a non-barbiturate sedative. The drug's mild side effects on the patient made it incredibly popular in the treatment of morning sickness. Two years later it was realized that thalidomide was causing a wide spectrum of very serious birth defects, and the drug was removed from the market [1-4]. Thalidomide most commonly induced birth defects in the limbs, and these defects ranged from amelia to phocomelia to digit loss $[3,5,6]$. Other thalidomide-induced defects included ear and eye problems, genital abnormalities, peripheral neuropathies, and internal organ defects (primarily to the kidneys, lungs, intestinal tract, and heart). Ultimately over 10,000 children worldwide were born with severe birth defects as a result of the teratogenic activities of thalidomide [2, 3, 7-10]. Despite its sordid history, thalidomide has reemerged as a treatment for multiple conditions including erythema nodosum leprosum, multiple myeloma, Crohn's Disease, and AIDS [11-17]. Unfortunately, this revival has been accompanied by a second generation of children with thalidomide-induced birth defects $[18,19]$, and highlighted the need for a complete understanding of the developmental mechanisms of thalidomide teratogenesis. In this study we develop a new model organism for the study of the cellular and molecular basis of thalidomide teratogenesis, the gray short-tailed opossum (Monodelphis domestica).

Despite its long and devastating history, the teratogenic mechanisms of thalidomide are not yet fully understood. Dozens of distinct theories for the mechanisms of thalidomide teratogenesis have been proposed, including DNA mutagenesis, disruption of chondrogenesis, nerve or neural crest toxicity, and inhibition of cell adhesion molecules [20, 21]. Currently, the most widely supported theories for thalidomide teratogenesis are based on the drug's abilities to disrupt angiogenesis [22-25]. The lack of resolution as to the mechanisms by which thalidomide generates human birth defects and the early misuse of the drug are primarily due to the complexity of the mechanisms themselves, and the lack of an appropriate mammalian model study system for thalidomide teratogenesis. Traditional mammal models, such as rats and mice, 
are resistant to the teratogenic properties of thalidomide. Though susceptible, studies of rabbit and other primates are hampered by the number of affected embryos that can be obtained. As a result, much of what is currently known about the mechanisms through which thalidomide acts has come from studies of zebrafish embryos, chicken embryos, or human umbilical vein endothelial cells (HUVECS) [22, 26-30]. Although research in these systems has provided important insights into thalidomide teratogenesis, these systems have limitations. Though zebrafish and chicken embryos display thalidomide induced appendage deformation, their possession of fins and wings rather than limbs limits what can be learned about the characteristic thalidomide limb deformities in humans [31]. HUVECS lack a developmental and cross-tissue context, and this limits what can be learned from them about the mechanisms by which thalidomide generates deformities in the embryo.

In this study we test the hypothesis that the gray short-tailed opossum (Monodelphis domestica) is well suited to model human thalidomide induced deformities. Opossums have large litters (up to 13 pups are born, through more embryos are sometimes present in the uterus), are easy to maintain and breed in the lab [32-34], have a fully sequenced and well-annotated genome [35], and are a well-established study system for development [35-42]. Opossums also display a unique mode of limb development in which the forelimbs grow at twice the rate of the hind limbs, which provides two temporal opportunities to study the impacts of teratogens on limb development $[34,43,44]$. Opossum forelimbs begin to grow out from the body at embryonic day (E) 10.75 , form a bud at E11.3, a paddle at E11.75, and by E13.75 the interdigital webbing has reduced and the digits are free. Opossum forelimbs therefore go from initial outgrowth to free digit formation in about 3 days. Opossum hind limbs first grow out from the body at E11.7, are buds at E12.2, and paddles shortly before birth at $\sim$ E14. The hind limbs complete their development after birth. For comparison purposes, mice limbs begin to grow out from the body at E9, form a bud at E11, a paddle at E12, and free digits at E15 - E16 [45]. However, despite the uniqueness of the timing of opossum limb development, most of the primary cellular and 
molecular drivers of limb development are highly conserved in opossum and other mammals $[36-38,45,47]$.

While the traits listed above make opossums a good model organism in general, for opossums to make a suitable mammalian model for thalidomide teratogenesis in humans they must be susceptible to thalidomide and display thalidomide-induced phenotypes that are similar to those observed in humans exposed to the drug. To evaluate whether opossums meet these criteria, we treated pregnant opossums with thalidomide and evaluated the impact of the thalidomide treatments on the morphology of the embryos.

\section{Methods}

Tissue Collection - All procedures described below have been approved by the University of Illinois IACUC. The Sears Lab (University of Illinois) maintains a breeding colony of opossums (Monodelphis domestica) using established protocols [32-34]. This colony is comprised of pedigreed animals from the Texas Biomedical Research Institute (San Antonio, TX) and their descendants. To generate timed matings, opossum males and females are placed together and recorded using infrared cameras [37]. The time at which mating occurs is defined as the time of conception.

Pregnant females were treated with a thalidomide (Sigma, $N=7$ ) or control solution $(\mathrm{N}=5)$ 10.75 days after conception through intraperitoneal (IP) injection, when embryos should be at Stage 26 (E10.75) [46, 47]. Stage 26 was selected for use because opossum organ development at this stage generally corresponds to the critical period of thalidomide sensitivity in humans, which is between 20 and 36 days after fertilization [7, 12, 48]. Thalidomide was delivered to each animal in solution at doses of $20 \mathrm{mg} / \mathrm{kg}$. As a point of reference, adult, female opossums generally weigh between 80 and 100 grams [31, 32]. The thalidomide solution contained the appropriate amount of thalidomide (for the body weight of the female) dissolved in phosphate 
buffered saline (PBS) with 1\% carboxymethyl cellulose and 1\% dimethyl sulfoxide (DMSO), and the control the same but without the thalidomide. A total of $100 \mu$ l of thalidomide solution was injected into each female. Females were monitored for overt, external signs of toxicity or stress (e.g., scruffy fur, loss of body weight, hunched posture, poor activity, difficulty breathing, shaking/shivering, dehydration, etc.). Pregnant females were euthanized by carbon dioxide inhalation to effect followed by cervical dislocation, and embryos dissected from the uterus and staged $[46,47]$.

To assess the morphology of thalidomide-exposed embryos, pregnant opossums were euthanized 3 days post-treatment at 13.75 days post-conception, shortly before birth (opossums are usually born between 14 and 15 days post-conception) [46]. Embryos dissected at this time should be at Stage $33[46,47]$. Embryos $(\mathrm{N}=60$ treatment, $\mathrm{N}=30$ control) were immediately examined and photographed using a Leica M205 C microscope and a Leica DFC425 digital microscope camera (Leica Microsystems Inc., Buffalo Grove, IL). While Stage 33 opossums are at an early stage of development (e.g., ossification has not yet begun), and a later developmental stage might be better for comparative purposes, the thalidomide-treated opossums do not usually survive after birth. While it is difficult to conclusively diagnose phocomelia at this early developmental stage (i.e., pre-ossification), many other birth defects are readily-apparent (e.g., blood vessel disruption, craniofacial defects, other limb defects, etc.).

\section{Results}

We did not observe any overt, external signs of toxicity or stress in the treated adult females. Three major phenotypes are present among the 60 thalidomide-treated embryos (from 7 litters) that were generated by this study. There was no evidence of any resorptions. We have classified these phenotypes as "normal", "mildly deformed", and "severely deformed." A breakdown of embryo numbers and phenotypes by litter is provided in Table 1. 
The external morphologies of embryos with the "normal" phenotype appear to have been unaffected by the thalidomide treatment. This phenotype class makes up $32 \%(\mathrm{~N}=19)$ of the treated embryos. Embryos from this class are full size, have normal proportions, and do not display any visible deformities (Figure 1A). In two "normal" embryos, the amniotic sac was constricted around the embryo just anterior to the hindlimb (e.g., amniotic band syndrome).

Most treated embryos, 57\% ( $=34)$, display the "mildly deformed" phenotype. "Mildly deformed" embryos occur in all treated litters (Figure 1B). These embryos are indistinguishable from control embryos in terms of overall size and limb patterning. Heartbeats can be visualized upon removal from the mother. However, all mildly deformed treated embryos present with at least one region of severe internal hemorrhaging and blood pooling, as was also seen in prior studies in monkeys [49]. These regions are commonly located near or in the fore- $(\mathrm{N}=14)$ or hind $(\mathrm{N}=9)$ limb, in the brain $(\mathrm{N}=17)$, or in the abdominal cavity $(\mathrm{N}=17)$ (Table 2$)$. In most regions, these hemorrhages tend to be asymmetrical. However, hemorrhaging in the region of the hind limbs tends to occur symmetrically. In about half of "mildly deformed" embryos $(\mathrm{N}=19)$, the hemorrhaging and blood pooling $(\mathrm{N}=19)$ is restricted to one general region (i.e., brain, foreor hind limb, abdominal cavity). In the other half, the hemorrhaging and blood pooling is present in two $(\mathrm{N}=10)$, three $(\mathrm{N}=4)$, or all four general regions $(\mathrm{N}=1)$. In two "mildly deformed" embryos, the amniotic sac was constricted around the embryo just anterior to the hindlimb (e.g., amniotic band syndrome). However, these two embryos did not display any signs of morphological deformation or size reduction.

Treated embryos displaying the "severely deformed" phenotype, $12 \%(\mathrm{~N}=7)$ exhibit a severely affected but variable external morphology. All treated embryos from this class are roughly or less than half the size of unaffected or mildly affected littermates, and all but one lacks pigmentation in the eye. As other physical traits vary among treated embryos, we describe each "severely deformed" treated embryo below. 
The least deformed of the "severely deformed" treated embryos (Embryo A) displays a novel amniotic sac defect (Figure 2A). Instead of a normal single amniotic sac, the amniotic sac has split into two chambers: one surrounding the embryo and one connecting to the chorionic plate. The embryo also displays significant hemorrhaging around its heart (Figure 2B). The heart itself is a small mass of improperly organized tissue beneath a single membrane, when it should be fully enclosed in the thoracic cavity by this point in development. While the cranial medial portion of the face appears to be normal above the jaw, there is no apparent mouth opening and the lateral portions of the cranium are missing or remarkably reduced in size. The forelimb is severely stunted, and displays a symmetrical loss of the first and fifth digits that results in a narrowed autopod. The hindlimb appears to be normal.

We observed similar amniotic defects, i.e., a dual-chambered amniotic sac, in another of the "severely deformed" treated embryos (Embryo B; Figure 2C). This embryo also has a large hemorrhage on the ventral surface around the heart, and its vascular networks appear immature with none of the major blood vessels evident (Figure 2D). The medial-rostral face of this embryo is also heavily deformed. Specifically, the medial portion of the mandibular process is severely reduced and the medial nasal process is completely absent. The cranial portion of the head also appears reduced in size. However, the limbs appear to be normally patterned despite the overall size reduction. Similar to Embryo A, this embryo appears to have been viable at least until the point of dissection as it has several traits that are characteristic of Stage 33 (E13.75) of opossum development (e.g., reduction in interdigital tissue, protruding tongue).

The remaining "severely deformed" treated embryos (Embryos C, D, E, F, and G) present none of the characteristics of Stage 33 (E13.75) of opossum development and most do not have visible blood flow. As a result, these embryos were not clearly viable at the time of dissection. Only one of these embryos, Embryo C (Figure 2E), has some minimal blood flow, although no major 
arteries are evident. However, the entire rostral portion of the cranium of Embryo $\mathrm{C}$ is greatly reduced, as is the region of embryo that is posterior to the forelimb. The digits of the forelimbs of Embryo $\mathrm{C}$ have also failed to differentiate properly and protrude at abnormal and asymmetrical angles.

Two "severely deformed" treated embryos from the same litter, Embryos D and E, display similar defects (D shown in Figure 2F). Neither embryo contains enough blood to visualize a vascular network. Though the faces of these embryos were damaged during dissection, both still clearly display a significantly reduced mandibular process. The hind limbs of both embryos are reduced in size and remain at an immature bud stage, similar to that observed at Stage 30 (E11.75) or 31 (E12.2) of opossum development [46]. The forelimbs of both embryos display an abnormal posterior orientation.

The forelimbs of another "severely deformed" treated embryo, Embryo F, are normal in their medial-lateral patterning (Figure 2G). They are, however, missing a single digit leaving the remaining digits in a symmetrical arrangement. The vascular network of Embryo $\mathrm{F}$ is also incomplete and disorganized.

The most disfigured of the treated embryos, Embryo G, is less than a third of the length of the "mildly deformed" and control embryos (Figure 2H and 2I). The vasculature of this embryo is limited to a few, very small pools of blood. Embryo G's limbs are also highly asymmetrical. The left limb resembles an enlarged version of the limb bud of a normal opossum at developmental Stage 28 [46]. The right limb is severely truncated in both width and length. Much of the cranial morphology of this embryo is unidentifiable due to gross deformity but the rostral region of the head appears to be absent or much reduced. 
This study also generated 30 control embryos from 5 litters for which the DMSO/CMC mixture used to dissolve thalidomide was injected alone. All embryos from four of the litters $(\mathrm{N}=25)$, and 2 embryos from the fifth display normal phenotypes (Figures 3A and 3B), resulting in 90\% of control embryos displaying normal phenotypes. Three embryos from the fifth litter, all of which were from a single uterine horn which was noted to be smaller than normal uterine horns at similar stages of pregnancy upon dissection, display some degree of malformation or developmental delay. The least deformed of these embryos exhibits mild blood pooling in the midbrain region and in small, isolated patches elsewhere on the body (e.g., forelimb, neck, back) (Figure 3C). While this embryo appears to display a midbrain blood pooling phenotype similar to that observed in 14 of the 60 "mild phenotype" thalidomide treated embryos described above, it does not display pooling in other regions in which pooling was commonly observed in "mild phenotype" treated embryos (e.g., forebrain in 9 embryos, on body ventral or dorsal to forelimb in 12 embryos). The other two control embryos are smaller in size, with one at $63 \%$ (Figure 3D) and the other $44 \%$ (Figure 3E) of the size of normal, control embryos. However, despite their size, both embryos display grossly normal limb phenotypes, with the larger displaying a paddlelike forelimb and bud-like hind limb similar to those of Stage 30 opossum embryos, and the smaller bud-like forelimbs and no perceivable hind limbs similar to Stage 28 embryos. Furthermore, neither embryo displays significant blood pooling or hemorrhaging, amniotic sac defects, heart defects, or perceivable mandibular defects.

\section{Discussion}

Embryonic opossums that have been exposed to thalidomide replicate many of the birth defects observed in humans that have been exposed to the drug $[2,3,5-8]$. These defects include limb truncations, digit losses, eye defects, craniofacial defects, developmental delays, reductions in overall size, vascular disruption, and heart defects. However, while we did observe limb truncations, we cannot conclusively state that any represent phocomelia because of the early developmental stages of the embryos (i.e., pre-ossification). 
The thalidomide-induced defects produced in this study differ from those produced in opossums exposed to retinoic acid, another teratogen, in a previous study (i.e., highly penetrant oligodactyly, exencephaly, no vascular disruption, etc.) [37]. In addition, control embryos generated in this study did not recapitulate several of the phenotypes observed in thalidomidetreated embryos, including severe and widely distributed blood pooling and hemorrhaging, limb truncations, digit losses, heart defects, and mandibular truncations. Taken together, these findings are consistent with these phenotypes being thalidomide-specific in opossums.

Thalidomide-exposed opossums also display phenotypes that have not previously been described in thalidomide-exposed animals, most notably amniotic sac defects. We observed amniotic sac constrictions both on and separate from the developing embryo after treatment with thalidomide. The relatively complete and normal development of the embryos inside some of these constrictions suggests that the constriction likely resulted from the fusion of the amniotic sac later in the pregnancy, rather than from defects in initial sac patterning. Alternatively, the constrictions could represent non-embryonated vesicles. While the cause of these amniotic sac defects remains unknown, the constriction we observed could be a cause of some of the less commonly seen thalidomide defects, such as the absence of the distal portions of the digits. In line with this, many of these less commonly observed thalidomide-induced defects in humans closely resemble those caused by the prenatal constrictions in amniotic band syndrome, so much so that the two disorders can be mistaken for each other [50].

The results of this study also support the hypothesis that thalidomide can severely disrupt angiogenesis in mammals. All embryos displaying a thalidomide-induced phenotype, be they "mildly deformed" or "severely deformed", exhibited clear angiogenic defects. These defects ranged from hemorrhages and/or blood pooling in "mildly deformed" embryos to a complete vascular disruption in some "severely deformed" embryos. Moreover, while one of control 
embryo exhibits some isolated blood pooling, and thus some evidence for angiogenic disruption, the range (i.e., structures impacted) and magnitude (i.e., degree of severity) of disruptions displayed by treatment embryos is greater. These findings are therefore consistent with the hypothesis that thalidomide induces defects in mammals, including humans, at least in part by disrupting proper angiogenesis $[21,22,51]$.

Unlike some other mammalian species that have been investigated $[52,53]$, thalidomide-induced birth defects are relatively easy to generate in opossums. Opossum embryos display a 60\% penetrance of the "mildly deformed" phenotype and a $12 \%$ penetrance of the "severely deformed" phenotype. This is consistent with human case reports, which estimate that approximately $20-50 \%$ of thalidomide exposed infants were born with abnormalities [3, 7, 48]. Given that the opossums from this study had an average of 7.5 embryos per litter, this means that most thalidomide-treated litters will likely produce many embryos displaying the "mildly deformed" phenotype and at least 1 embryo with the "severely deformed" phenotype. In line with this, all treated litters in our study generated several "mildly deformed" and at least 1 “severely deformed” embryo.

To confirm the results obtained from the limited trials in this study, additional thalidomide and control trials should be performed in the opossum system. However, given the opossum's litter size, high penetrance of thalidomide-induced defects, and defects similar to humans, results of this initial study support the hypothesis that the gray short-tailed opossum (Monodelphis domestica) makes a good mammalian model system for thalidomide-induced deformities in humans. In particular, the opossum system seems to provide a good opportunity to investigate the hypothetical role of vascular disruption in thalidomide teratogenesis. Furthermore, while the reproductive process of opossums, as marsupial mammals, does differ from that of humans and other placental mammals in some ways (e.g., choriovitelline placenta in marsupials vs. hemochorial placenta in humans), both marsupial and placental mammals share internal 
development, a reproductive trait critical to the process of teratogenesis. This similarity represents a significant advantage of the opossum model, relative to in vitro and non-mammalian models. We are following up on the research presented here by performing additional thalidomide and control trials in opossum, and detailed analyses of the cellular and molecular processes that are disrupted by thalidomide exposure in the opossum model.

\section{Acknowledgements}

We thank the members of the Sears Lab and J. Marcot for discussion of ideas presented in this manuscript, and the DAR at the University of Illinois for insights into animal husbandry. We also thank the Suarez Lab for the use of equipment. This research was supported by National Science Foundation (1257873) and University of Illinois Research Board (16056) grants to K.E.S. 
Table 1. Phenotypes of treated and control embryos at Stage 33, by litter.

\begin{tabular}{|c|c|c|c|c|c|c|c|}
\hline & $\begin{array}{l}\text { Total } \\
\text { embryos }\end{array}$ & $\begin{array}{l}\text { Normal } \\
\text { Phenotype }\end{array}$ & $\begin{array}{l}\% \\
\text { Normal }\end{array}$ & $\begin{array}{l}\text { Mild } \\
\text { Phenotype }\end{array}$ & $\begin{array}{l}\% \\
\text { Mild }\end{array}$ & $\begin{array}{l}\text { Severe } \\
\text { Phenotype }\end{array}$ & $\begin{array}{l}\% \\
\text { Severe }\end{array}$ \\
\hline Treated Litter 1 & 12 & 4 & 33 & 6 & 50 & 2 & 17 \\
\hline Treated Litter 2 & 13 & 3 & 23 & 9 & 69 & 1 & 8 \\
\hline Treated Litter 3 & 9 & 1 & 11 & 8 & 89 & 0 & 0 \\
\hline Treated Litter 4 & 6 & 3 & 50 & 3 & 50 & 0 & 0 \\
\hline Treated Litter 5 & 10 & 4 & 40 & 5 & 50 & 1 & 10 \\
\hline Treated Litter 6 & 5 & 1 & 20 & 4 & 80 & 0 & 0 \\
\hline Treated Litter 7 & 5 & 1 & 20 & 1 & 20 & 3 & 60 \\
\hline Control Litter 1 & 10 & 10 & 100 & 0 & 0 & 0 & 0 \\
\hline Control Litter 2 & 4 & 4 & 100 & 0 & 0 & 0 & 0 \\
\hline Control Litter 3 & 3 & 3 & 100 & 0 & 0 & 0 & 0 \\
\hline Control Litter 4 & 8 & 8 & 100 & 0 & 0 & 0 & 0 \\
\hline Control Litter 5 & 5 & 2 & 40 & 1 & 20 & 2 & 40 \\
\hline
\end{tabular}


Table 2. Sites of hemorrhages and/or pooled blood in embryos displaying the "mildly deformed" phenotype. Embryos with multiple hemorrhages are listed in more than one row in upper section of table. $\%$ of treated embryos is out of the total number of thalidomide-treated embryos, $\mathrm{N}=60$.

Location

BRAIN

Forebrain

Midbrain

Brain stem

ABDOMINAL

FORELIMB ASSOCIATED

On body, just ventral to forelimb

On body, just dorsal to forelimb

Upper and fore- arm

Hand

HIND LIMB ASSOCIATED

Upper and fore- leg

Foot

Co-occurrence of locations

ONE LOCATION

Brain

Abdominal

Forelimb

Hind limb

TWO LOCATIONS

Brain + abdominal

Brain + forelimb

Brain + hind limb

Abdominal + forelimb

Abdominal + hind limb

Forelimb + hind limb

THREE LOCATIONS

Brain + abdominal + forelimb

Brain + abdominal + hind limb

Brain + forelimb + hind limb

Abdominal + forelimb + hind limb

FOUR LOCATIONS
Number of embryos \% of treated displaying phenotype embryos $(\mathrm{N}=60)$

0

0

1

0 
Figure 1. Both embryos in this figure were exposed to thalidomide at Stage 26 and dissected at Stage 33. The embryo on the left (A) does not display any phenotype, i.e., is from the "normal" phenotype class, and that on the right (B) is from the "mildly deformed" class. Most embryos that have been exposed to thalidomide display a "mildly deformed" phenotype, similar to the embryo in (B), that is characterized by hemorrhaging and blood pooling in various regions of the embryo. Arrows indicate patches of blood pooling.

Figure 2. Several thalidomide-exposed embryos display "severely deformed" phenotypes, but the phenotypes of these embryos vary. Shown here are the amniotic sac phenotype (A) and anatomy (B) of Embryo A, the amniotic sac phenotype (C) and anatomy (D) of Embryo B, and the anatomy of Embryos C (E), D (F), E (G), and F (H, I). All scale bars represent $0.25 \mathrm{~mm}$.

Figure 3. Most control embryos $(\mathrm{N}=22,90 \%)$ display a "normal" phenotype (A, B). Three control embryos, all coming from one uterine horn of a single litter, display either blood pooling (C) or smaller than normal sizes (D and E). However, none of the control embryos display severe and widely distributed blood pooling and hemorrhaging, limb truncations, digit losses, heart defects, or mandibular truncations. 


\section{References}

[1] Gillis L. Thalidomide babies: management of limb defects. BMJ. 1962;2:647-51.

[2] Lenz W. A short history of thalidomide embryopathy. Teratology. 1988;38:203-15.

[3] Lenz W, Knapp K. Foetal malformations due to thalidomide. German Med Monthly. $1962 ; 7: 253-8$.

[4] Vargesson N. Thalidomide embryopathy: An enigmatic challenge. ISRN Dev Biol. 2013;2013:1-18.

[5] McBride MS. Thalidomide and congenital abnormalities. Lancet. 1961;2:1358.

[6] Leck IM, Millar EL. Incidence of malformations since the introduction of thalidomide. BMJ. $1962 ; 2: 16-20$.

[7] Smithells RW, Newman CG. Recognition of thalidomide defects. J Med Genet. 1992;29:71623.

[8] Newman CG. Clinical observations on the thalidomide syndrome. Proc R Soc Med. 1977;70:225-7.

[9] Newman CG. The thalidomide syndrome: risks of exposure and spectrum of malformations. Clin Perinatol. 1986;13:555-73.

[10] Ruffing L. Evalulation of thalidomide children. Birth Defects Orig Artic Ser. 1977;13:287300 .

[11] Calabrese L, Fleischer AB. Thalidomide: current and potential clinical applications. Am J Med. 2000;108:487-95.

[12] Miller MT, Stromland K. Teratogen update: thalidomide: A review, with a focus on ocular findings and new potential uses. Teratology. 1999;60:306-21.

[13] Franks ME, Macpherson GR, Figg WD. Thalidomide. Lancet. 2004;363:1802-11.

[14] Bartlett JB, Dredge K, Dalgleish AG. The evolution of thalidomide and its IMiD derivatives as anticancer agents. Nat Rev Cancer. 2004;4:314-22.

[15] Teo SK, Stirling DI, Zeldis JB. Thalidomide as a novel therapeutic agent: new uses for an old product. Drug Discov Today. 2005;10:107-14. 
[16] Teo SK. Properties of thalidomide and its analogues: implications for anticancer therapy. AAPS J. 2005;7:E14-9.

[17] Galustian C, Labarthe MC, Bartlett JB, Dalgleish AG. Thalidomide-derived immunomodulatory drugs as therapeutic agents. Expert Opin Biol Ther. 2004;4:1963-70.

[18] Castilla EE, Ashton-Prolla P, Barreda-Mejia E, Brunoni D, Cavalcanti DP, Correa-Neto J, et al. Thalidomide, a current teratogen in South America. Teratology. 1996;54:273-7.

[19] Schuler-Faccini L, Soares RC, de Sousa AC, Maximino C, Luna E, Schwartz IV, et al. New cases of thalidomide embryopathy in Brazil. Birth Defects Res A Clin Mol Teratol. 2007;79:6712.

[20] Vargesson N. Thalidomide-induced teratogenesis: history and mechanisms. Birth Defects Res C Embryo Today. 2015;105:140-56.

[21] Vargesson N. Thalidomide-induced limb defects: resolving a 50-year-old puzzle. BioEssays. 2009;31:1327-36.

[22] Therapontos C, Erskine L, Gardner ER, Figg WD, Vargesson N. Thalidomide induces limb defects by preventing angiogenic outgrowth during early limb formation. Proc Natl Acad Sci U S A. $2009 ; 106: 8573-8$.

[23] Ito T, Ando H, Handa H. Teratogenic effects of thalidomide: molecular mechanisms. CMLS. 2011;68:1569-79.

[24] Stephens TD. The effect of thalidomide in chicken embryos. Birth Defects Res A Clin Mol Teratol. 2009;85:725-31.

[25] D'Amato RJ, Loughnan MS, Flynn E, Folkman J. Thalidomide is an inhibitor of angiogenesis. Proc Natl Acad Sci U S A. 1994;91:4082-5.

[26] Yabu T, Tomimoto H, Taguchi Y, Yamaoka S, Igarashi Y, Okazaki T. Thalidomideinduced antiangiogenic action is mediated by ceramide through depletion of VEGF receptors, and is antagonized by sphingosine-1-phosphate. Blood. 2005;106:125-34.

[27] Knobloch J, Shaughnessy JD, Jr., Ruther U. Thalidomide induces limb deformities by perturbing the Bmp/Dkk1/Wnt signaling pathway. FASEB J. 2007;21:1410-21. 
[28] Tamilarasan KP, Kolluru GK, Rajaram M, Indhumathy M, Saranya R, Chatterjee S.

Thalidomide attenuates nitric oxide mediated angiogenesis by blocking migration of endothelial cells. BMC Cell Biol. 2006;7:17.

[29] Beedie SL, Rore HM, Barnett S, Chau CH, Luo W, Greig NH, et al. In vivo screening and discovery of novel candidate thalidomide analogs in the zebrafish embryo and chicken embryo model systems. Oncotarget. 2016;7:33237-45.

[30] Mahony C, Erskine L, Niven J, Greig NH, Figg WD, Vargesson N. Pomalidomide is nonteratogenic in chicken and zebrafish embryos and nonneurotoxic in vitro. Proc Natl Acad Sci U S A. 2013;110:12703-8.

[31] Ito T, Ando H, Suzuki T, Ogura T, Hotta K, Imamura Y, et al. Identification of a primary target of thalidomide teratogenicity. Science. 2010;327:1345-50.

[32] Keyte AL, Smith AJ. Basic maintenance and breeding of the opossum Monodelphis domestica. Cold Spring Harb Protoc. 2008.

[33] Rousmaniere H, Silverman R, White RA, Sasaki MM, Wilson SD, Morrison JT, et al. Husbandry of Monodelphis domestica in the study of mammalian embryogenesis. Lab Anim (NY). 2010;39:219-26.

[34] Keyte AL, Smith KK. Opossum (Monodelphis domestica): A marsupial developmental model. Cold Spring Harb Protoc. 2008.

[35] Mikkelsen TS, Wakefield MJ, Aken B, Amemira CT, Chang JL, Duke S, et al. Genome of the marsupial Monodelphis domestica reveals innovation in non-coding sequences. Nature. 2007;447:167-77.

[36] Sears KE, Maier JA, Rivas-Astroza M, Poe R, Zhong S, Kosog K, et al. The relationship between gene network structure and expression variation among individuals and species. PLoS Genet. 2015;11:e1005398.

[37] Molineaux AC, Maier JA, Schecker T, Sears KE. Exogenous retinoic acid induces digit reduction in opossums (Monodelphis domestica) by disrupting cell death and proliferation, and 
apical ectodermal ridge and zone of polarizing activity function. Birth Defects Res A Clin Mol Teratol. 2015;103:225-34.

[38] Beiriger A, Sears KE. Cellular basis of differential limb growth in postnatal gray shorttailed opossums (Monodelphis domestica). J Exp Zool B Mol Dev Evol. 2014;322:221-9. [39] Hubler M, Molineaux AC, Keyte A, Schecker T, Sears KE. Development of the marsupial shoulder girdle complex: a case study in Monodelphis domestica. Evol Dev. 2013;15:18-27. [40] Samollow PB. The opossum genome: Insights and opportunities from an alternative mammal. Genome Res. 2008;18:1199-215.

[41] Vaglia JL, Smith KK. Early differentiation and migration of cranial neural crest in the opossum, Monodelphis domestica. Evol Dev. 2003;5:121-35.

[42] Morrison JT, Bantilan NS, Wang VN, Nellett KM, Cruz YP. Expression patterns of Oct4, Cdx2, Tead4, and Yap1 proteins during blastocyst formation in embryos of the marsupial, Monodelphis domestica. Evol Dev. 2013;15:171-85.

[43] Doroba CK, Sears KE. The divergent development of the apical ectodermal ridge in the marsupial Monodelphis domestica. Anat Rec. 2010;293:1325-32.

[44] Keyte AL, Smith KK. Developmental origins of precocial forelimbs in marsupial neonates Development. 2010;137:4283-94.

[45] Wanek N, Muneoka K, Holler-Dinsmore G, Burton R, Bryant SV. A staging system for mouse limb development. J Exp Zool. 1989;249:41-9.

[46] Mate KE, Robinson ES, Vandeberg JL, Pedersen RA. Timetable of in vivo embryonic development in the grey short-tailed opossum (Monodelphis domestica). Mol Reprod Dev. 1994;39:365-74.

[47] McCrady E. The embryology of the Opossum. Philadelphia: Wistar Institute of Anatomy and Biology; 1938.

[48] Lenz W, Knapp K. Thalidomide embryopathy. Arch Environ Health. 1962;5:100-5.

[49] Delahunt C, Lassen L. Thalidomide syndrome in monkeys. Science. 1964;146:1300-5. 
[50] Goldfarb CA, Sathienkijkanchai A, Robin NH. Amniotic constriction band: a multidisciplinary assessment of etiology and clinical presentation. J Bone Joint Surg. 2009;91:68-75.

[51] Vargesson N, Hootnick DR. Arterial dysgenesis and limb defects: clinical and experimental examples. Reprod Toxicol. in press.

[52] Nudleman KL, Travill AA. A morphological and histochemical study of thalidomideinduced upper limb malformations in rabbit fetuses. Teratology. 1971;4:409-25.

[53] Giroud A, Tuchmann-Duplessis H, Mercier-Parot L. [Observatios on the teratogenic repercussions of thalidomide in the mouse and rabbit]. C R Seances Soc Biol Ses Fil. 1962;156:765-8. 
Figure 1
Click here to download high resolution image

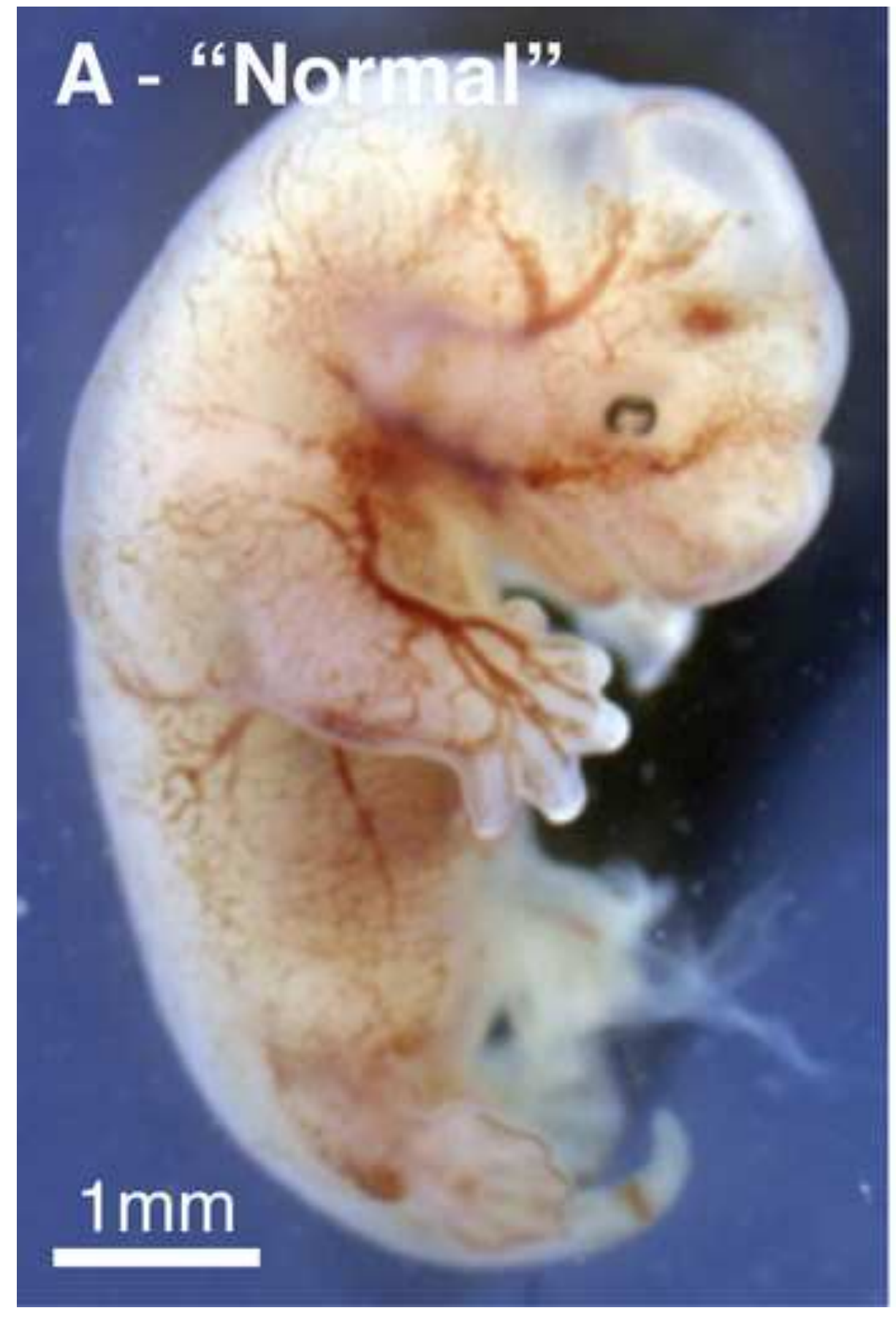

B - "Mildly deformed"

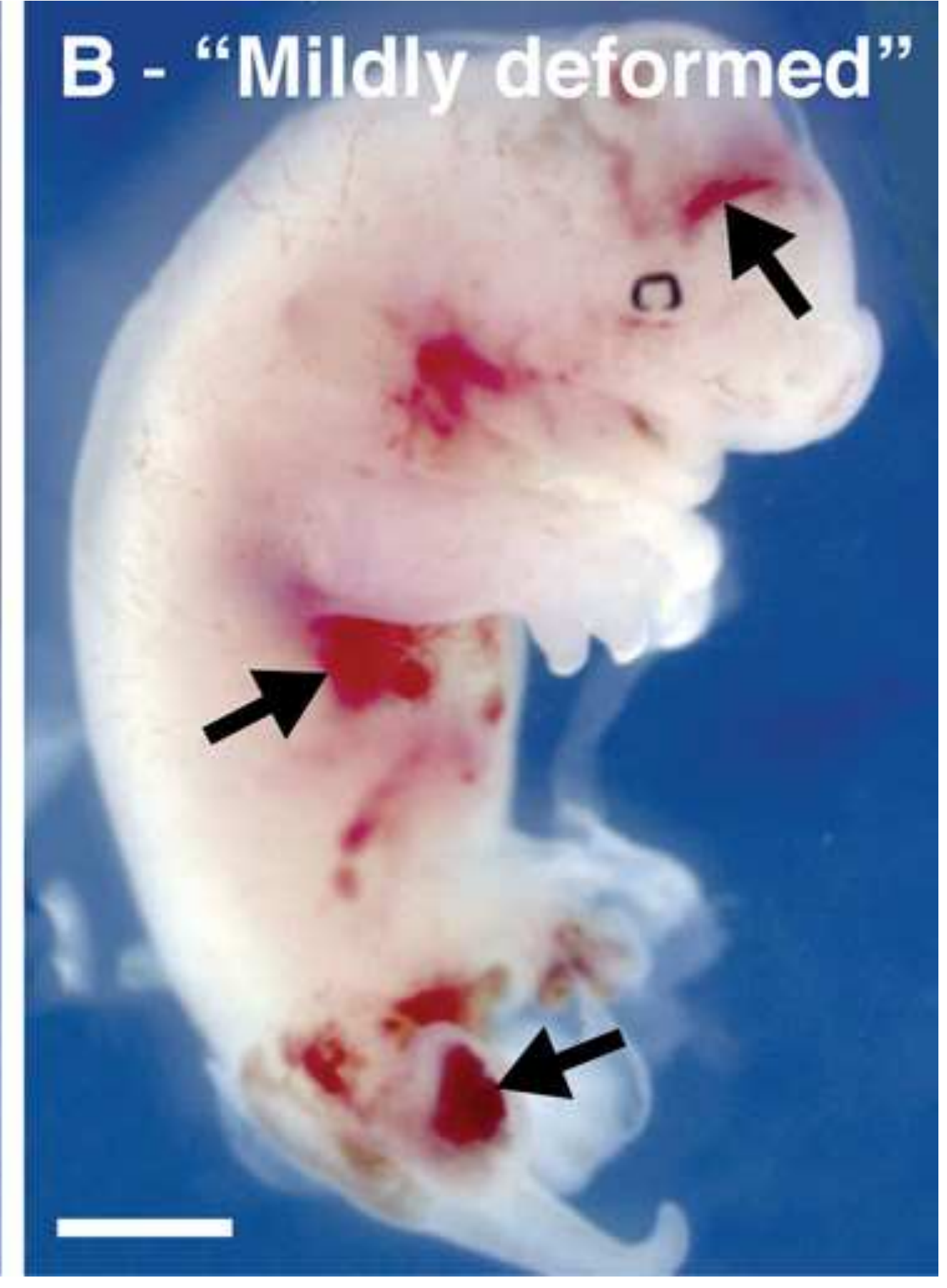

Figure 1
Click here to download high resolution image
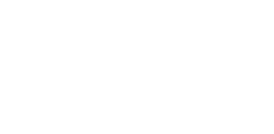

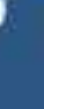




\section{Click here to download high resolution image}
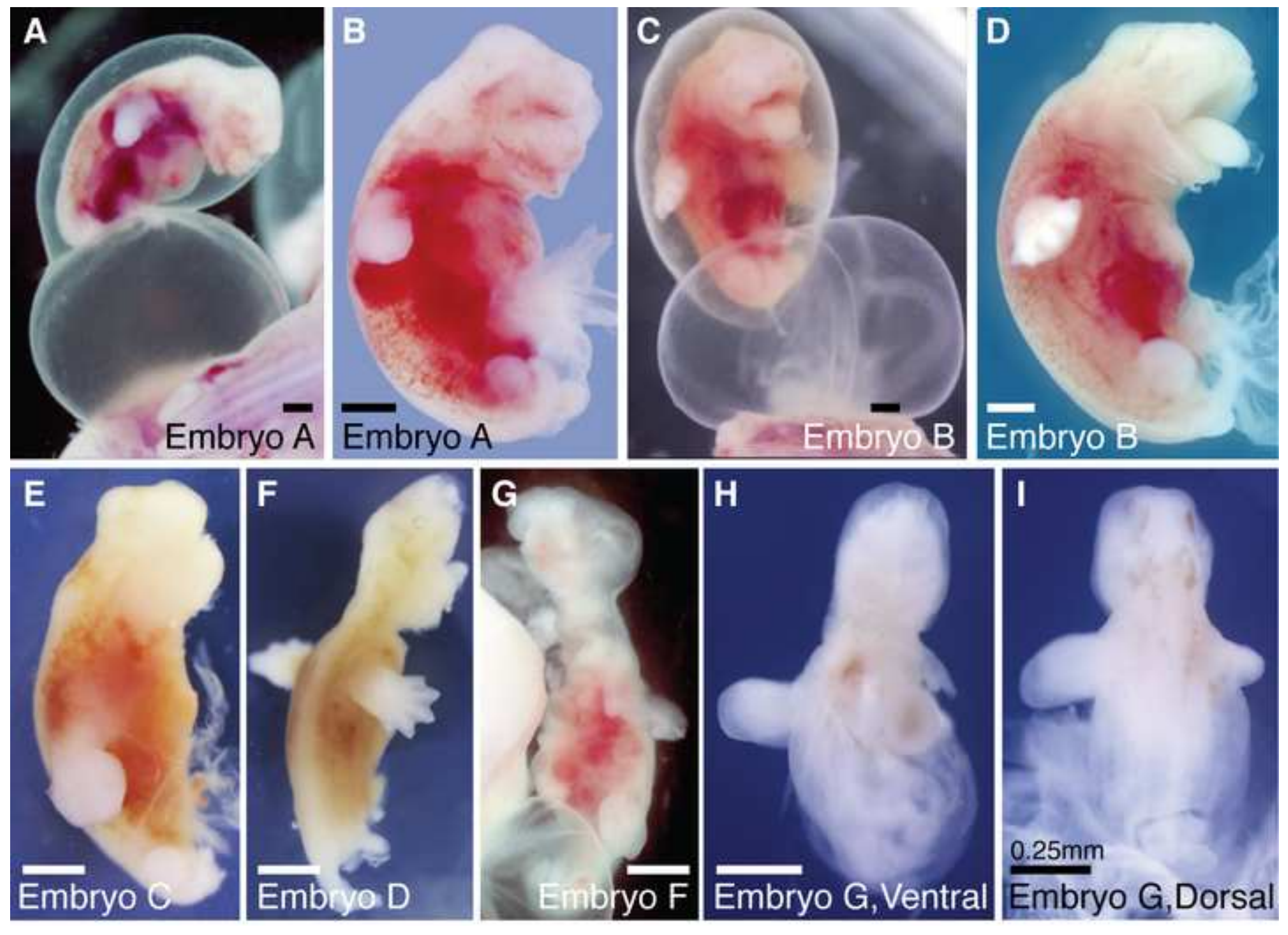

Embya D

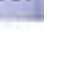


Click here to download high resolution image
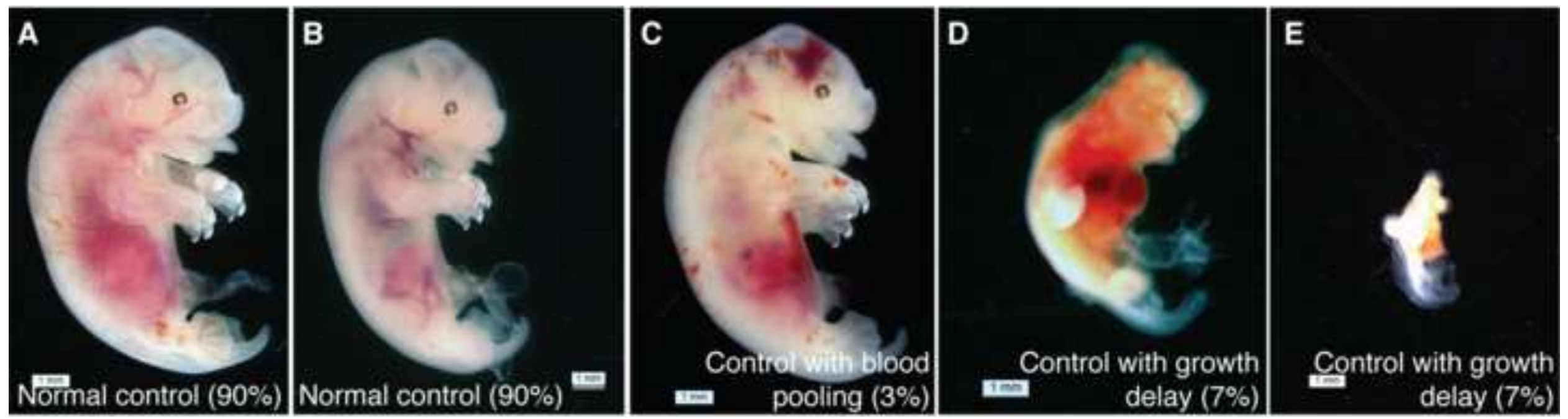
${ }^{*}$ Conflict of Interest Statement
Click here to download Conflic

Click here to download Conflict of Interest Statement: Sorensen_coi_disclosure.pdf 